\title{
Synthesis of copper-ferrous $(\mathrm{CuFe})$ nanowires via electrochemical method and its investigations as a fluid sensor
}

\author{
SALEEM KHAN ${ }^{1}$, SANDEEP ARYA ${ }^{1}$, PARVEEN LEHANA ${ }^{1}$ and SURESH KUMAR ${ }^{2, *}$ \\ ${ }^{1}$ Department of Physics \& Electronics, University of Jammu, Jammu 180 006, India \\ ${ }^{2}$ Department of Electronic Science, Kurukshetra University, Kurukshetra 136 119, India
}

MS received 29 April 2013; revised 15 June 2013

\begin{abstract}
The special behaviour of nanowires with respect to electrical conductivity makes them suitable for sensing application. In this paper, we present a copper-ferrous ( $\mathrm{CuFe}$ ) nanowires based sensor for detection of chemicals. CuFe nanowires were synthesized by template-assisted electrochemical method. By optimizing the deposition parameters, continuous nanowires on a copper substrate were synthesized. The morphological and structural studies of the synthesized CuFe nanowires were carried out using scanning electron microscope (SEM) and X-ray diffraction (XRD). Substrates containing CuFe nanowires were moulded to form a capacitor. Different chemicals were used as dielectric in the capacitor which showed that the capacitance was a nonlinear function of the dielectric constant of fluid unlike the linear relation shown by conventional capacitors. This unique property of the nanowires based capacitors may be utilized for developing fluid sensors with improved sensitivity.
\end{abstract}

Keywords. Electrochemical deposition; nanowires; nanosensor; dielectric.

\section{Introduction}

A better understanding of the fundamental properties of matter in reduced dimensions has been contributed by the development of nanotechnology (Sordan et al 2001). Studies of nanomaterials are of great interest due to their potential applications in many fields of science and technology (Wilson and Gifford 2005). Depending upon the dimensions, nanomaterials are divided into four categories; 0D, 1D, 2D and 3D nanostructured materials. Out of these four nanostructured materials, 1D nanostructures, commonly exist in the form of nanowires and nanotubes and are extensively explored because of their wide range of applications in microelectronics, medicine, biology, catalysis and sensing technology. The scope of this paper is limited to only nanosensors.

Sensors are composed of an active sensing material with a signal transducer. These components in sensors transmit the signal without any amplification from a selective compound or from a change in a reaction. Sensors produce signals in the form of electrical, thermal or optical variations. These signals may be converted to digital signals for further processing (Konishi et al 2003; Wilson and Gifford 2005; Yogeswaran and Chen 2008). Both nanowires and nanotubes can function as sensing element and the electrical contact that accesses them. These are the primary building blocks of the nanosensors (Myung

*Author for correspondence (sawan2k2@yahoo.co.in) et al 2004). High surface-to-volume ratio makes nanowires and nanotubes excellent candidates for sensing applications. High surface-to-volume ratio improves detection sensitivity and response time due to more reaction area per volume and reduced diffusion time (Grujicic and Pesic 2002; Xie 2002; Huang et al 2004).

Nanowires can be fabricated by various techniques that include lithographic patterning (Rahman et al 2009; Bandaru and Pichanusakorn 2010), vapour transport techniques (Bae et al 2004; Malandrino et al 2004), templatebased synthesis methods (Martin 1996; Kline et al 2006), and other synthesis methods (Xu et al 2002; Zheng et al 2005). The template-based synthesis involving electrochemical deposition is more promising owing to its specific advantages of low cost and control over the nanowire properties by changing the electrolyte composition, $\mathrm{pH}$, temperature and applied potential or the current (Chakarvarti 2006). Today, various techniques are being used for the fabrication of 1D nanostructures, but the template synthesis is a versatile and economic one for synthesizing a variety of $1 \mathrm{D}$ nanomaterials including metals, semiconductors, heterojunctions, conducting polymers, CNTs and much more (Singh et al 2006). Metallic nanowires synthesized by template-assisted electrodeposition method have been proved to be a cheap and high yield technique (Hamrakulov et al 2009). Nuclear array glasses, mesoporous channel hosts, polycarbonate membrane and selfordered anodized aluminum oxide (AAO) films are used as templates in electrodeposition process for synthesizing nanowires. Track-etch polycarbonate nanopore membrane 
(Whatman) was used as template in this to synthesize 1D nanostructures of copper-ferrous. The size and spacing of nanofeatures can be controlled using templates with different characteristics (Huang et al 2006; Chen et al 2008; Arya et al 2013). Nanowires are mechanically stable within the templates and easy to handle. In conclusion, the template based electrodeposition method for the fabrication of nanowires has proved to be a reliable and effective technique.

The objective of this paper is to investigate the applications of electrochemically-deposited CuFe nanowires as fluid sensors. The investigations carried out included various fluids such as water $\left(\mathrm{H}_{2} \mathrm{O}\right)$, acetone $\left(\mathrm{C}_{3} \mathrm{H}_{6} \mathrm{O}\right)$, ethanol $\left(\mathrm{C}_{2} \mathrm{H}_{5} \mathrm{O}\right)$, dichloromethane $\left(\mathrm{CH}_{2} \mathrm{Cl}_{2}\right)$ and chloroform $\left(\mathrm{CHCl}_{3}\right)$. The methodology of the investigations was carried out and is presented in the next section. Results and conclusions are presented, thereafter.

\section{Experimental}

The experimental set up used for investigating the applications of electrochemically deposited nanowires is divided into two phases. In the first phase, the nanowires are synthesized using a two-electrode system. The cell consists of a cylindrical container mounted over one side of a disc-shaped acrylic sheet with a strong adhesive. An orifice of precise diameter is made in the disc along the axis of the cylinder and a ring-shaped rubber cork is attached to it. This arrangement is placed over a metallic plate which completes the cell system. Two electrodes are used in the experiment; a platinum electrode acting as anode and self-adhesive copper tape on metallic circular sheet as cathode. The mechanism of deposition process for nanowires is shown in figure 1 . A $100 \mathrm{~nm}$ pore sized polycarbonate membrane is placed over copper tape substrate which acts as a sacrificial layer.

The copper substrate along with the membrane is placed in contact with metallic plate, which serves as the cathode. The growth of nanowires by electrochemical process was achieved by applying a d.c. voltage of $0 \cdot 8 \mathrm{~V}$ across the plating bath, which is filled with the electrolytic solution of $0.2 \mathrm{M}$ of $\mathrm{CuSO}_{4} \cdot 6 \mathrm{H}_{2} \mathrm{O}, 0.2 \mathrm{M}$ of $\mathrm{FeSO}_{4} \cdot 7 \mathrm{H}_{2} \mathrm{O}$ and $0 \cdot 1 \mathrm{M}$ of $\mathrm{H}_{3} \mathrm{BO}_{3}$ prepared in $50 \mathrm{ml}$ of double-distilled water. $\mathrm{pH}$ of the electrolyte was 2.9 in this experiment. The electrodeposition was carried out at room temperature for 12 min duration. The length of the nanowires was controlled by the duration of the electrodeposition.

The polycarbonate membrane was dissolved in dichloromethane to release the nanowires. Change in current density during deposition was monitored and it was observed that at initial stage of the electrodeposition process, current density had high value and started decreasing, reaching a constant region, where deposition of nanowires occurred and after that over-deposition process took place. The structural properties were studied by X'PERT-PRO Phillips X-ray diffractometer using $\mathrm{CuK} \alpha$ radiation at $45 \mathrm{~mA}$ and $45 \mathrm{keV}$. The morphologies of CuFe nanowires were investigated by JEOL JSM-6100 analytical scanning electron microscope (SEM).

\section{Results and discussion}

\subsection{SEM characterization}

The surface morphology of CuFe nanowires have been studied by scanning electron microscopy. The dried sample were mounted on aluminum stub with the help of adhesive carbon tape and coated with a layer of gold in JEOL JFM 1100 Sputter Coater and then viewed under scanning electron microscope. Figures 2 and 3 show surface morphology (top view) of grown CuFe nanowires of
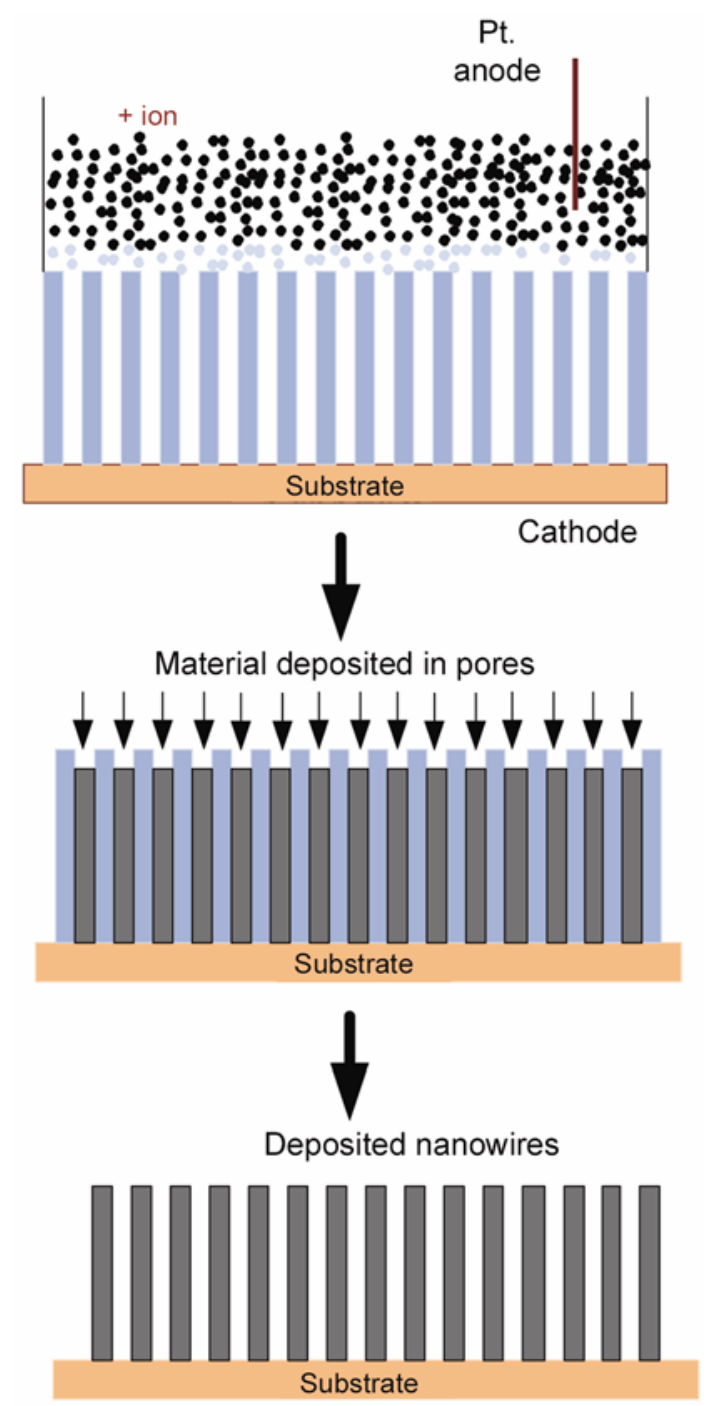

Figure 1. Schematic diagram showing mechanism of nanowires synthesis via electrochemical method. 
diameters $100 \mathrm{~nm}$ with zooming $X=6500$ and $X=9000$, respectively.

SEM studies show that the growth nanowires are uniform and have diameter equal to the diameter of the pores of polycarbonate template used. The electrochemically synthesized $\mathrm{CuFe}$ nanowires are found to be highly ordered, vertically aligned and of high aspect ratio. SEM image of a copper-ferrous mixed metal nanowire is fabricated using a polycarbonate membrane. It may be observed from SEM that mixed metal nanowires of copper and ferrous have been successfully fabricated. The diameter of the nanowires was fixed by the actual pore size of the polycarbonate membrane, while the length of the nanowires was varied based on time duration of the applied voltage.

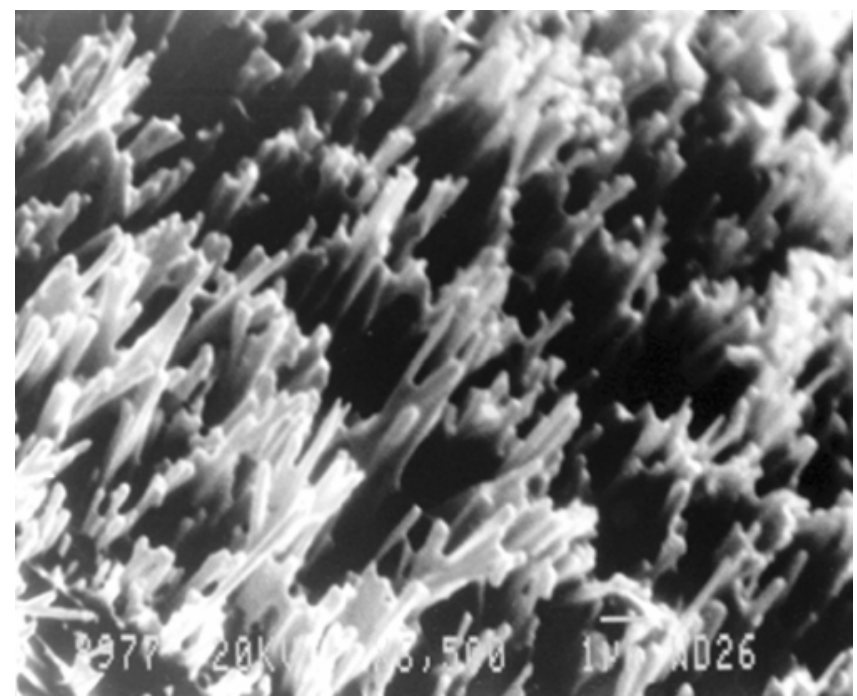

Figure 2. SEM of CuFe nanowires $(100 \mathrm{~nm})$ deposited on copper substrate with $X=6500$.

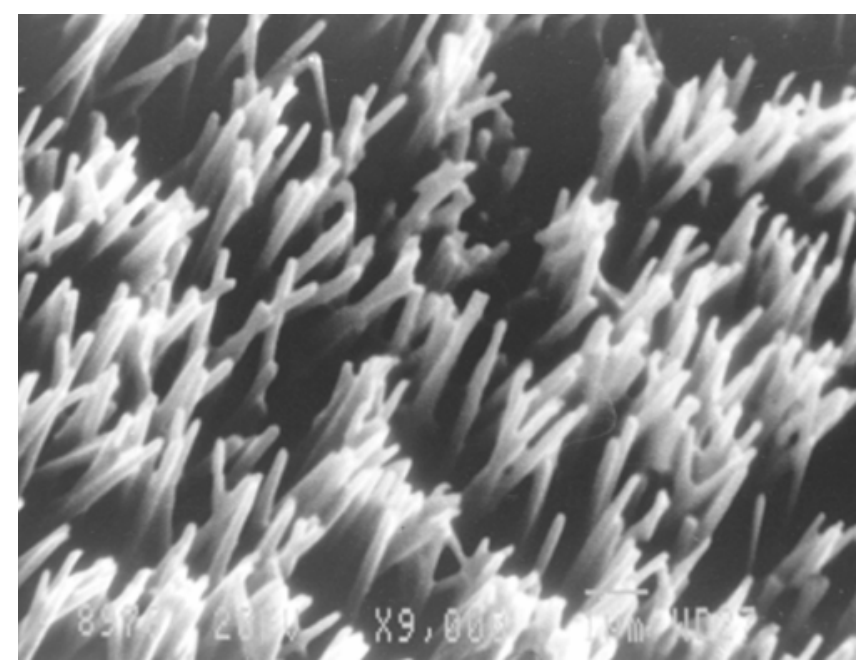

Figure 3. SEM of CuFe nanowires $(100 \mathrm{~nm})$ deposited on copper substrate with $X=9000$.

\subsection{XRD characterization}

Figure 4 shows XRD pattern of electrochemically synthesized CuFe nanowire arrays of diameter $100 \mathrm{~nm}$ on copper substrate. XRD patterns were recorded in the range of scanning angle, $10-70^{\circ}$ with a step size of $0.0170^{\circ}$ using wavelength $(K \alpha), 1.5406 \AA$. From the XRD studies, it is found that the $\mathrm{CuFe}$ nanowires are determined to have cubic structure since the characteristic diffraction peaks in the diffraction spectrum of CuFe nanowires match with the standard ICDD copper ferrous $\left(\mathrm{Cu}_{x} \mathrm{Fe}_{1-x}\right)$ data file (JCPDS number: 49-1399). XRD studies confirm the formation of $\mathrm{CuFe}$ nanowires.

Various XRD peaks of CuFe from (111), (220), (311) and (333) planes are observed at $2 \theta$ angle of $17.89^{\circ}$, $29.61^{\circ}, 31.69^{\circ}$ and $56.52^{\circ}$ for $100 \mathrm{~nm}$ nanowires, respectively. Two strong peaks of copper are also observed in

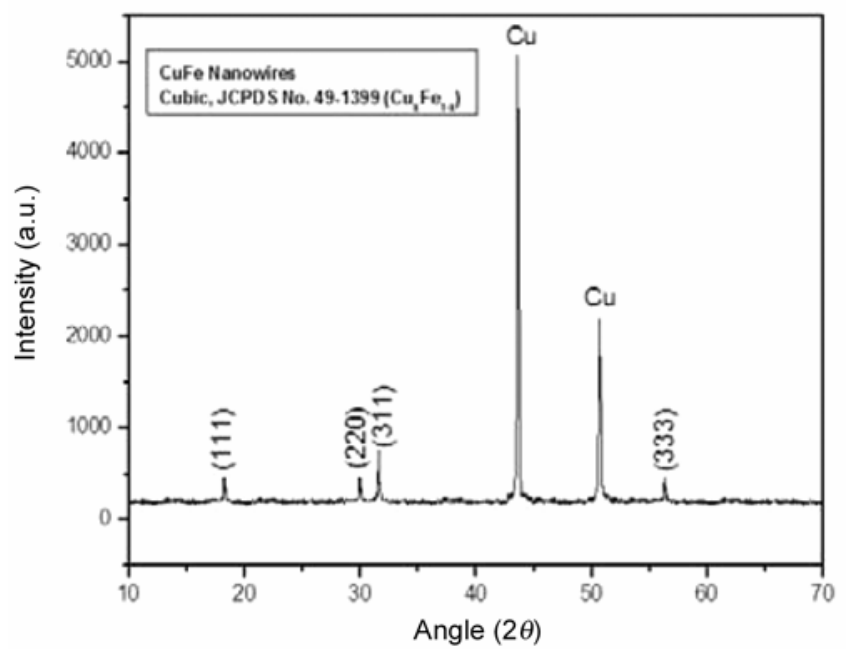

Figure 4. XRD pattern of CuFe nanowires $(100 \mathrm{~nm})$ on copper substrate.

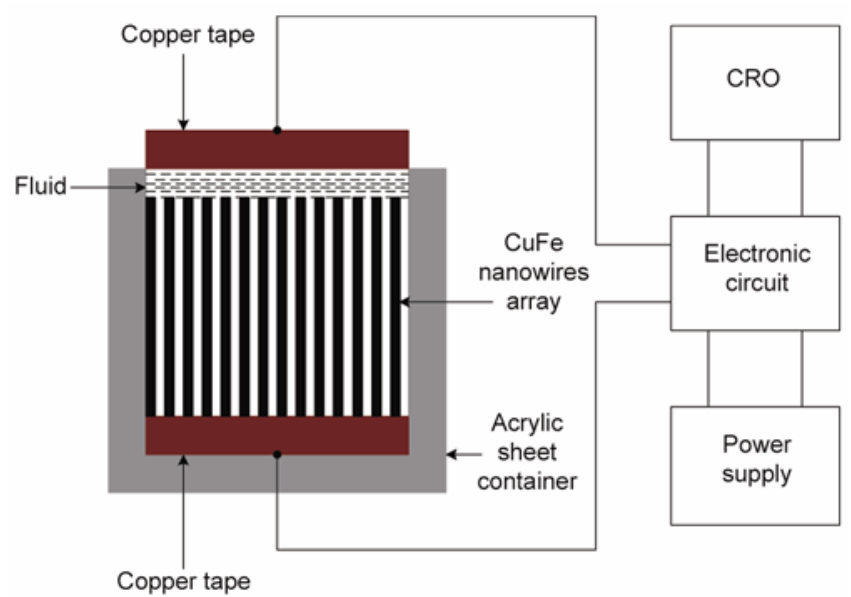

Figure 5. Block diagram implementing $\mathrm{CuFe}$ nanowires in electronic circuit. 
the XRD pattern which arises from the copper substrate. XRD peaks of CuFe shows crystalline nature of as-grown $\mathrm{CuFe}$ nanowires. The diffraction peaks of $100 \mathrm{~nm} \mathrm{CuFe}$ nanowires were also observed to be broadened as compared to bulk material. This broadening of the diffraction peaks is due to nanometer size of nanowires.

\subsection{CuFe nanowire as fluid sensor}

In order to use $\mathrm{CuFe}$ nanowires as sensor in electronic circuitry, a capacitor is made from the substrate containing
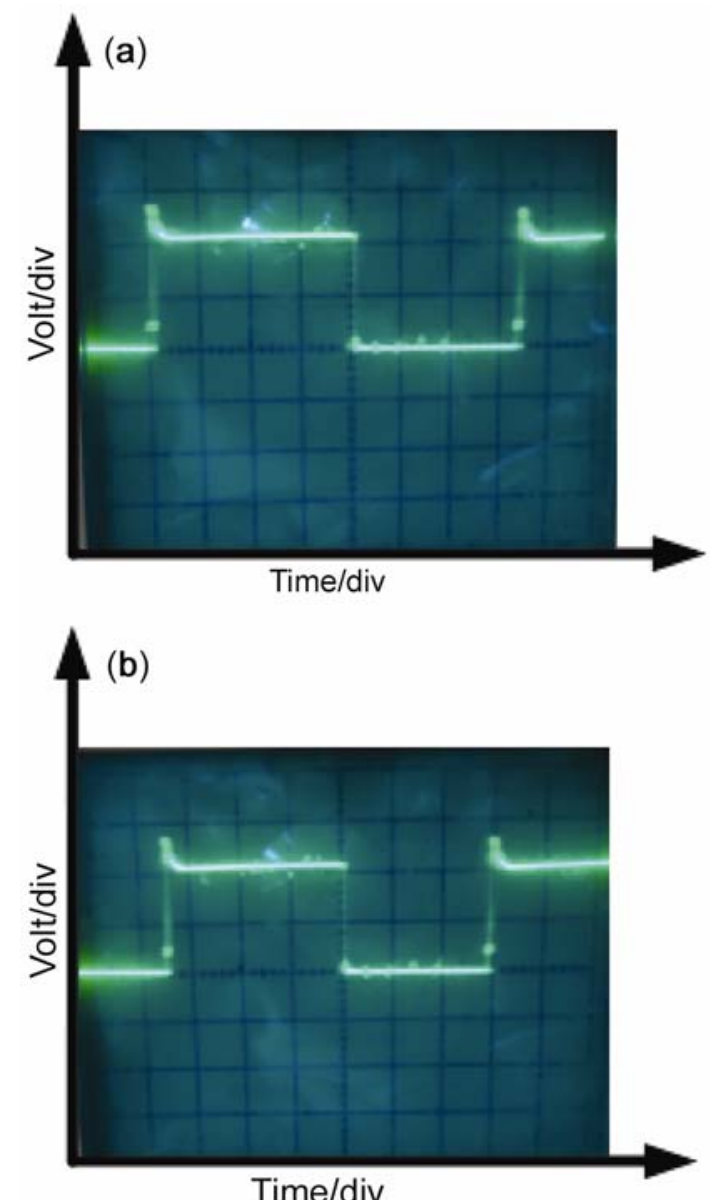

Figure 6. Depending upon dielectric, change induced in time period of a stable multivibrator: (a) $\mathrm{H}_{2} \mathrm{O}$ and (b) air, dielectric recorded on CRO.

Table 1. Calculated capacitor parameters.

\begin{tabular}{lccc}
\hline Fluid & $\begin{array}{c}\text { Dielectric } \\
\text { value }\end{array}$ & $\begin{array}{c}\text { Oscillation } \\
\text { time }(\mu \mathrm{s})\end{array}$ & Capacitance $(\mathrm{pF})$ \\
\hline $\mathrm{H}_{2} \mathrm{O}$ & 80 & $3 \cdot 7$ & 11.865 \\
$\mathrm{C}_{3} \mathrm{H}_{6} \mathrm{O}$ & 21.5 & $2 \cdot 1$ & 6.73 \\
$\mathrm{C}_{2} \mathrm{H}_{5} \mathrm{O}$ & $16 \cdot 2$ & $2 \cdot 7$ & 8.65 \\
$\mathrm{CH}_{2} \mathrm{Cl}_{2}$ & $8 \cdot 93$ & $1 \cdot 4$ & 4.48 \\
$\mathrm{CHCl}_{3}$ & $4 \cdot 8$ & 1.7 & $5 \cdot 45$ \\
\hline
\end{tabular}

CuFe nanowires. The schematic of the circuit implementing capacitor made from substrate containing CuFe nanowires is shown in figure 5 . The capacitor is used in a stable mode of multi-vibrator electronic circuit (Karris 2008). The capacitor parameters such as charging time, discharging time and oscillation time period were estimated from the CRO. Capacitance values with different dielectric medium were calculated from the following formulae:

$$
\begin{aligned}
& T_{\mathrm{d}}=0.693 R_{\mathrm{B}} C, \\
& T_{\mathrm{C}}=0.693\left(R_{\mathrm{A}}+2 R_{\mathrm{B}}\right) C,
\end{aligned}
$$

where $R_{\mathrm{A}}=10 \mathrm{k} \Omega$ and $R_{\mathrm{B}}=220 \mathrm{k} \Omega$. Total time period of oscillations is estimated from CRO by visualizing the output waveform, which is given as

$$
T=T_{\mathrm{c}}+T_{\mathrm{d}} \text {. }
$$

The values of capacitances were calculated for various chemical fluids taking them as a dielectric medium between the plates of CuFe nanowires-based capacitor. The dielectrics were chosen in increasing order of dielectric constant. The fluid chosen for dielectric medium are water $\left(\mathrm{H}_{2} \mathrm{O}\right)$, acetone $\left(\mathrm{C}_{3} \mathrm{H}_{6} \mathrm{O}\right)$, ethanol $\left(\mathrm{C}_{2} \mathrm{H}_{5} \mathrm{O}\right)$, dichloromethane $\left(\mathrm{CH}_{2} \mathrm{Cl}_{2}\right)$ and chloroform $\left(\mathrm{CHCl}_{3}\right)$ having dielectric values $80,21 \cdot 5,16 \cdot 2,8.93$ and $4 \cdot 8$, respectively at room temperature.

Table 1 shows estimated values of the total oscillation time period for these fluids. It was observed that on changing the dielectric in between the plates of the capacitor, duration of the 'ON' and 'OFF' states of a stable multivibrator was changed (figure 6). Although, it seems very common, the amount of change is not predictable from the basic formula of the capacitance.

These results are further emphasized in figure 7 . It shows that the change in capacitance of $\mathrm{CuFe}$ nanowires based capacitor as sensor is not directly proportional to the dielectric constant of the fluid; hence the behaviour is not similar to a conventional capacitor.

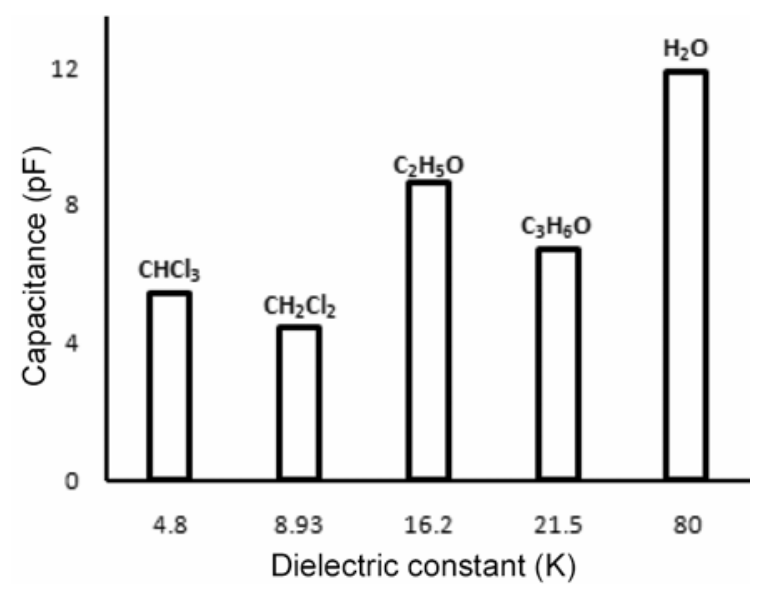

Figure 7. Calculated values of capacitance of CuFe nanowires capacitive sensor with increasing order of dielectric value. 
CuFe noanowires show different capacitive behaviours toward the dielectric material used in the moulded capacitor. This property of CuFe nanowires may be used for fabrication of fluid sensors which can be further investigated to characterize different fluids in the environment.

\section{Conclusions}

The dielectric-dependent capacitive properties of $\mathrm{CuFe}$ nanowires of diameter $100 \mathrm{~nm}$, synthesized electrochemically via template synthesis using polycarbonate tracketch membrane have been studied and reported. SEM studies have confirmed that the CuFe nanowires are ordered, vertically aligned and of high aspect ratio. Capacitance measurements of $\mathrm{CuFe}$ nanowires embedded in polycarbonate membrane have shown nonlinear characteristics. The analysis of the results of using copper substrate along with the deposited $\mathrm{CuFe}$ nanowires as one plate of a parallel plate capacitor showed that the change in the capacitance is different from the predicted value using simple classical formula. These results may be very useful for developing a fluid sensor.

\section{Acknowledgements}

This work is financially supported by the University Grants Commission, New Delhi, India, under plan for financial support for major research project grant no. F. No. 3455/2008 (SR).

\section{References}

Arya S, Khan S, Kumar S, Verma R and Lehana P 2013 BOMS-D-12-00212 (in press)

Bae S Y, Seo H W and Park J H 2004 J. Phys. Chem. B108 5206
Bandaru P R and Pichanusakorn P 2010 Semicond. Sci. Tech. 25

Chakarvarti S K 2006 Proc. SPIE 617

Chen X, Duan H, Zhou Z, Liang J and Gnanaraj J 2008 Nanotechnology 19365306

Grujicic D and Pesic B 2002 Electrochim. Acta 472901

Hamrakulov B, Kim I S, Lee M G and Park B H 2009 Trans. Nonferrous Met. Soc. China 19 s83

Huang J, Virji S, Weiller B H and Kaner R B 2004 Chem. Eur. J. 101314

Huang Q, Davis D and Podlaha E J 2006 J. Appl. Electrochem. 36871

Karris S T 2008 Electronics devices and amplifier circuits: with MATLAB applications (Fremont, California: Orchad Publication) 2nd edn

Kline T R, Tian M, Wang J, Sen A, Chan M W H and Mallouk T E 2006 Inorg. Chem. 457555

Konishi Y, Motoyama M, Matsushima H, Fukunaka Y, Ishii R and Ito Y 2003 J. Electro-anal. Chem. 559149

Malandrino G, Finocchiaro S T, Nigro R L, Bongiorno C, Spinella C and Fragala I L 2004 Chem. Mater. 1655

Martin C R 1996 Chem. Mater. 81739

Myung N V, Yun M and Vasquez R P 2004 Nano Lett. 4 419

Rahman S F A, Hashim U, Nor M N, Nuri A M, Shohini M E A and Salleh S 2009 AIP Conf. Proc. 1136504

Singh R, Kumar R, Sharma S K and Chakarvarti S K 2006 Dig. J. Nanomater. Biostruct. 1149

Sordan R, Burghard M and Kern K 2001 Appl. Phys. Lett. 79 13

Wilson G S and Gifford R 2005 Biosens. Bioelectron. 20 2388

Xie D, Jiang Y, Pan W, Li D, Wu Z and Li Y 2002 Sensor Actuat. B81 158

Xu C, Xu G, Liu Y and Wang G 2002 Solid State Commun. 122 175

Yogeswaran U and Chen S 2008 Sensors 8290

Zheng D, Sun S, Fan W, Yu H, Fan C, Cao G, Yin Z and Song X 2005 J. Phys. Chem. B109 16439 